\title{
Evaluation of Rigid and Non-rigid Motion Compensation of Cardiac Perfusion MRI
}

\author{
Hui Xue ${ }^{1,2,3}$, Jens Guehring ${ }^{1}$, Latha Srinivasan ${ }^{2}$, Sven Zuehlsdorff ${ }^{4}$, Kinda Saddi ${ }^{1}$, \\ Christophe Chefdhotel ${ }^{1}$, Joseph V. Hajnal ${ }^{2}$, and Daniel Rueckert ${ }^{3}$ \\ ${ }^{1}$ Imaging and Visualization, Siemens Corporate Research, Princeton, NJ, USA \\ ${ }^{2}$ Imaging Sciences Department, Imperial College, London, Du cane Road, W12 0NN, UK \\ ${ }^{3}$ Department of Computing, Imperial College, London, 180 Queen's Gate, SW7 2BZ, UK \\ ${ }^{4}$ MR Research and Development, Siemens Medical Solutions USA, Inc., Chicago, IL, USA \\ \{hui.xue, 1.srinivasan, d.rueckert, jo.hajnal\}@imperial.ac.uk, \\ \{jens.guehring, sven.zuehlsdorff, kinda.saddi, \\ christophe. chefdhotel\} asiemens.com
}

\begin{abstract}
Although the evaluation of cardiac perfusion using MRI could be of crucial importance for the diagnosis of ischemic heart diseases, it is still not a routinely used technique. The major difficulty is that MR perfusion images are often corrupted by inconsistent myocardial motion. Although motion compensation methods have been studied throughout the past decade, no clinically accepted solution has emerged. This is partly due to the lack of comprehensive validation. To address this deficit we collected a large multi-centre MR perfusion dataset and used this to characterize typical myocardial motion and confirmed that under clinically relevant conditions motion correction is a frequent requirement (67\% of all 586 cases). We then developed a proposed solution which includes both rigid/affine and the non-rigid image registration. Quantitative validation has been conducted using 6 different statistics to provide a comprehensive evaluation, showing the proposed techniques to be highly robust to different myocardial anatomy and motion patterns as well as to MR imaging acquisition parameters.
\end{abstract}

\section{Introduction}

The evaluation of myocardial perfusion is of crucial importance in the diagnosis and therapeutic decision-making of known and suspected ischemic heart disease. Different techniques have been developed to measure coronary flow and myocardial perfusion. Although nowadays only radionuclide imaging techniques, such as Single Photon-Emission Computed Tomography (SPECT) and Positron-Emission Tomography (PET) are widely used in the clinical setting [1], myocardial MR perfusion imaging is becoming popular. Perfusion MR imaging offers several advantages including its non-invasive nature, the ability to achieve higher spatial resolution and the flexible imaging orientation.

Despite clear advantages over radionuclide techniques, MR perfusion imaging is still not routinely used by cardiologists and radiologists. One major difficulty is the perfusion evaluation using $\mathrm{MR}$ is often undermined by inconsistent myocardial 
motion mainly caused by patient breathing and imperfect cardiac gating. As a result a motion compensation procedure must be applied prior to compute the myocardial signal intensity (SI) curves during the first or second pass of the injected MR contrast agent.

To maximize the clinical applicability, an effective motion compensation method should be able to cope with different MR pulse sequences which are now used to assess MR perfusion. Clinically three widely used sequences are TurboFLASH (Turbo Fast Low Angle Shot), TrueFISP (True Fast Imaging with Steady state Precession), and GRE-EPI (Gradient Echo type Echo Planar Imaging) [1,2]. Although all three can be classified as MR sequences, they can produce perfusion images with variant spatial resolution, myocardial contrast and signal-to-noise ratio (SNR), which challenges the motion compensation method. Moreover, clinical routine requires the sufficient coverage of the entire left ventricle (LV), so that a large part of myocardium can be imaged. However, due to the limited imaging efficiency of current MR scanners, the MR perfusion sequences are basically two-dimensional (2D) and thus multislice imaging techniques must employed. Generally, a minimum of three short-axis slices coving the basal, medial and apical part of LV is recommended [1,3], as shown in Fig. 1. Further increasing the number of slices does not significantly improve the assessment of myocardial perfusion [3]. The motion compensation method is therefore required to cope with different myocardial anatomy and align perfusion series for all three different slice positions.

Different motion compensation algorithms have been proposed to correct MR perfusion series. The majority of them focuses to compensate the rigid-body motion using image registration [4-10], while an exception is recently published [11] with the ICA (independent component analysis) being used to analyze the myocardium intensity patterns and to estimate the 2D translation. To correct the possible non-rigid deformation of myocardium during the contrast uptake, methods based on active contour [12] and active shape model [13] have been developed.

Despite the above-mentioned methodology studies throughout the past decade, no clinically accepted solution has emerged. This is partly due to lack of comprehensive validation. To the best of our knowledge, none of these proposed techniques have been explicitly validated on the large clinical datasets including all main perfusion MR pulse sequences and different slice positions (basal, medial and apical).

To address this deficit we collected a large multi-center MR perfusion dataset covering typical clinical configurations and used this to first characterize typical myocardial motion and then to develop a proposed solution which includes both rigid/affine
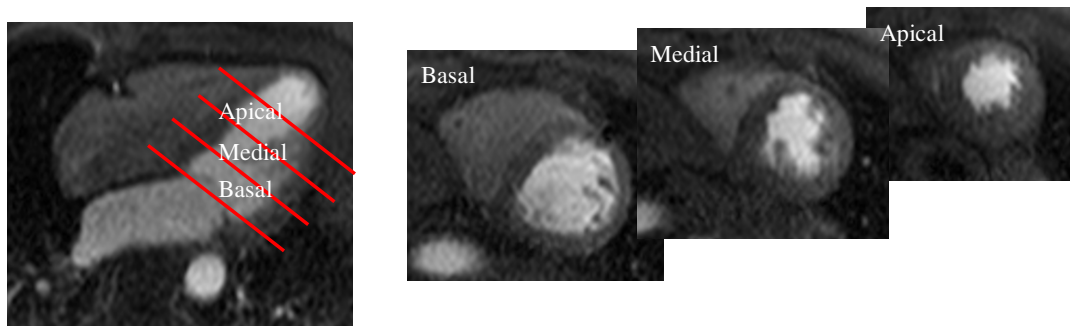

Fig. 1. To cover the entire LV, a minimum of three short-axis slices are required 
and the non-rigid image registration. Quantitative validation has been conducted using 6 different statistics to provide a comprehensive evaluation, showing the proposed techniques to be highly robust to different myocardium motion, slice positions and MR imaging acquisition parameters.

\section{Materials and Methods}

\subsection{Datasets}

Data from 76 patients scanned in 4 different institutes (CCB, Frankfurt, Germany; Elisabeth-Krankenhaus, Essen, Germany; NHLBI, Bethesda, MD, USA; and the Royal Brompton Hospital, London, UK) was collected, with a total of 586 image series, including 28,241 2D frames. Three different MR perfusion imaging sequences (TurboFLASH, TrueFISP, and GRE-EPI) were used in these scans. All scans were performed with a minimum of three slice positions (basal, medial and apical).

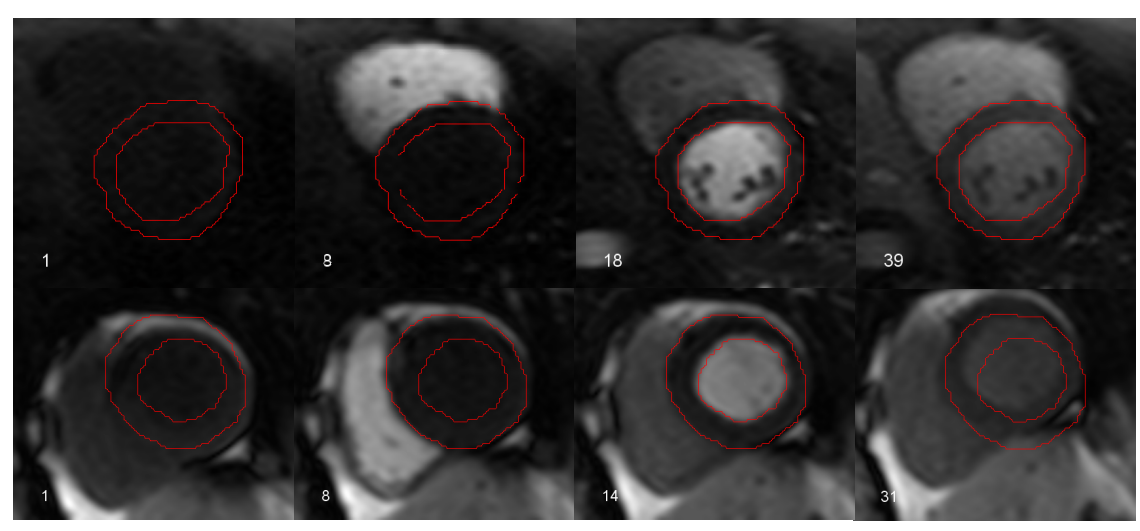

Fig. 2. An illustration of different myocardium motion. First row: the GRE-EPI perfusion series does not show discernible motion. Second row: this TrueFISP image shows significant motion.

To verify the necessity for motion compensation in the clinical setting, we visually reviewed all datasets and classified them into two categories (no significant motion and with significant motion) according to the maximal motion magnitude presented in the series. No discernible motion was found in 196 series (33\%) while the other 390 series $(67 \%)$ clearly require motion compensation, which emphasized the necessity of the motion compensation for MR perfusion assessment. As an illustration, two typical perfusion series are shown in Fig. 2.

The motion corrupted perfusion sequences (390 in total) were further analyzed to determine the patterns of myocardium motion. In 122 series the myocardium shows no visible local deformation and a rigid transformation (translation and rotation) is therefore adequate. The other 268 series displayed discernible non-rigid deformation of the myocardium, suggesting the use of non-rigid registration for these cases. Note that no data was excluded from the qualitative analysis, or from the evaluation of motion correction procedures, maximizing the fidelity to the real clinical setting. 


\subsection{Rigid/Affine Motion Compensation}

A general rigid/affine registration framework is implemented, including image transformation, interpolator, similarity metric and optimizer. We have achieved a highly robust rigid/affine registration for MR perfusion series by adopting a multi-level optimizer which is defined as a container of a specific single-step optimizer. Within this scheme, the step-size of the optimization algorithm is reduced by a factor of two after every optimization step. The multi-level optimizer is used with the multiresolution image pyramid to enlarge the capture range. An outline of the multiresolution multi-level rigid/affine registration algorithm is shown in Algorithm 1.

As a practical motion compensation algorithm requires the robustness to different configurations, we fixed the parameters of the rigid and affine registration and used them to align all perfusion series. For the rigid registration, three resolution levels and five optimization level were used (both were scaled by 2), while two resolution levels and six optimization levels were adopted for affine registration. The single-level optimizer was based on the simple downhill algorithm. For all perfusion series, the rigid registration was performed fist and its output was fed into the affine registration as the initial transformation.

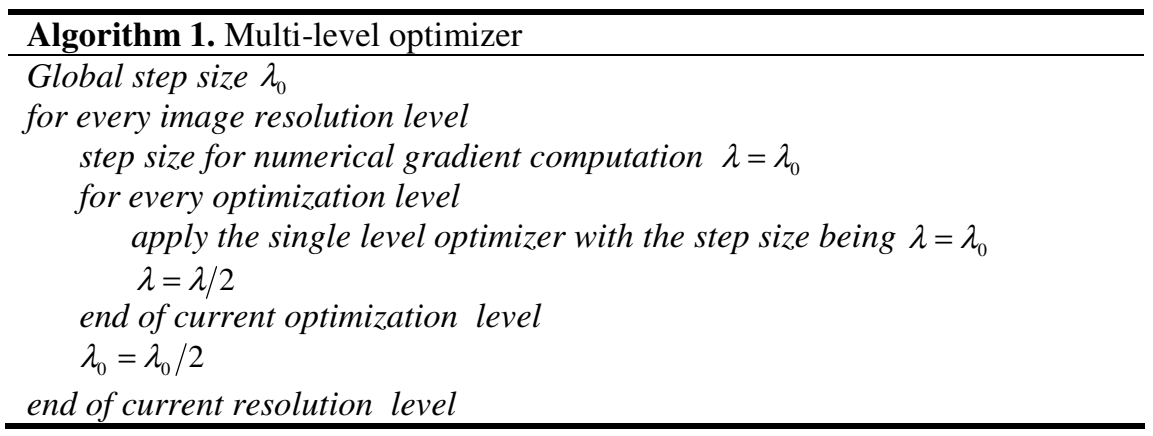

\subsection{Non-rigid Motion Compensation}

A Free-Form Deformation (FFD) based non-rigid registration algorithm [14] is applied after the rigid/affine motion correction. In this framework, a dense 2D deformation field is parameterized at a sparse control point lattice. The motion vector at every control point is estimated to optimize the cost function. A multi-level B-spline FFD transformation is used to allow non-uniform control point lattice spacing and facilitate the capture of large deformation fields, as suggested in [15].

To define a FFD for the myocardium and left ventricle, we define the spatial domain occupied by heart as follows: $\Omega_{s}=\{(x, y, z) 0 \leq x \leq X, 0 \leq y \leq Y, 0 \leq z \leq Z\}$ and $\phi_{s}$ denotes a $n_{x} \times n_{y}$ grid of control points $\varphi_{i, j}$. The spacing between adjacent control points is uniform in all coordinate directions. The deformation of a $2 \mathrm{D}$ spatial point $v_{i}=(x, y, z)$ is represented as the $2 \mathrm{D}$ tensor of the 1-D cubic B-splines:

$$
\boldsymbol{T}_{\text {local }}\left(\boldsymbol{v}_{i}\right)=\sum_{m=0}^{3} \sum_{n=0}^{3} B_{m}(u) B_{n}(v) \varphi_{i+l, j+m}
$$


where $i=\left\lfloor x / n_{x}\right\rfloor-1, j=\left\lfloor y / n_{y}\right\rfloor-1, u=x / n_{x}-\left\lfloor x / n_{x}\right\rfloor$, and $v=y / n_{y}-\left\lfloor y / n_{y}\right\rfloor \cdot B_{m}$ represents the $\mathrm{m}$-th basis function of the B-spline. The basis functions of cubic Bsplines have limited support. Therefore changing a control point in the grid affects only a $4 \times 4$ region around that control point. To ensure that the spatial transformation defined by the FFD is smooth and the unrealistic deformation of myocardium is minimized, the standard second order regularization penalty should be added into the similarity metric [14].

Again, tunable parameters were empirically fixed for all non-rigid registration. The two levels B-spline FFD was used and the control point spacing was $30 \mathrm{~mm}$ for the coarse level and halved for the finer level. The weighting coefficient to balance the regularization penalty is 0.0025 for both FFD levels.
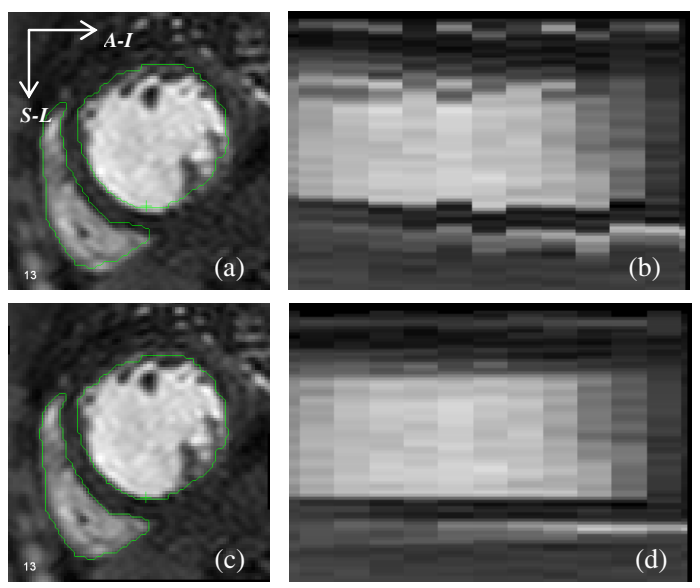

Fig. 3. An example of MR perfusion motion compensation. Before registration: (a) A 2D slice overlaid with myocardium contour extracted from the template slice; (b) The intensity-time relationship for this series. After motion compensation: (c) the same slice as (a) and (d) the corrected intensity-time relationship. This image was acquired using the GRE-EPI pulse sequence.

For both rigid/affine and non-rigid motion correction, normalized mutual information (NMI) [16] is maximized during the registration, as the MR perfusion series commonly show large intensity changes within myocardium and LV during the contrast agent passage. A slice selected for peak signal change during first pass of the contrast in the myocardium is used as a template and all other slices are transformed into the template coordinate system. We found the registration to be more robust if the slices to be aligned have similar contrast, even when NMI is used. Therefore, registration is performed consecutively between temporally adjacent slices, starting from the template and its direct neighbors (previous and next). For the rigid/affine registration, the computed transformation matrices are easily concatenated while for the non-rigid registration, a slice is registered to its warped neighbors that have been transformed into the template coordinate system. 


\section{Experimental Approaches}

Registration performance is first evaluated by means of visual inspection. Specifically, each data set is assessed visually and graded by an expert. For the 196 series classified as 'no significant motion', two grades are used (failed and same). A corrected series is classified as 'failed', if the registration introduces unwanted jitter, given the original series does not present discernible motion. The grade 'same' means the registration does preserve the quality of original series. For the 390 series with significant motion, the results of motion compensation should clearly decrease the jitter of myocardium. The three grades used here are then 'failed, not improved and improved'. The grade 'not improved' means there are still clear motion left after the compensation and further processing is required, while 'improved' means the significant motion throughout the whole series has been largely cured.

Table 1. The quantitative measures for the perfusion series

\begin{tabular}{|c|c|c|c|c|c|c|c|c|c|c|}
\hline & \multicolumn{3}{|c|}{$T_{A-I}[\mathrm{~mm}]$} & \multicolumn{3}{|c|}{$T_{S-L}[\mathrm{~mm}]$} & \multicolumn{3}{|c|}{ Dice ratio } \\
\hline & & no & $\mathbf{r} / \mathbf{a}$ & ffd & no & $\mathbf{r} / \mathbf{a}$ & ffd & no & r/a & ffd \\
\hline \multirow{4}{*}{ 之े } & Basal & 0.713 & 0.713 & 0.442 & 0.359 & 0.359 & 0.653 & 0.942 & 0.942 & 0.940 \\
\hline & Medial & 0.761 & 0.758 & 0.507 & 0.361 & 0.353 & 0.438 & 0.922 & 0.921 & 0.909 \\
\hline & Apical & 0.391 & 0.391 & 0.574 & 0.660 & 0.660 & 0.807 & 0.906 & 0.906 & 0.888 \\
\hline & Total & 0.621 & 0.621 & 0.507 & 0.460 & 0.457 & 0.633 & 0.923 & 0.923 & 0.912 \\
\hline \multirow{4}{*}{ 路 } & Basal & 4.006 & 0.893 & 0.745 & 6.925 & 1.210 & 0.837 & 0.783 & 0.926 & 0.935 \\
\hline & Medial & 4.027 & 1.122 & 0.625 & 6.291 & 0.644 & 0.521 & 0.674 & 0.890 & 0.913 \\
\hline & Apical & 2.314 & 1.487 & 0.943 & 7.172 & 1.257 & 1.197 & 0.674 & 0.882 & 0.894 \\
\hline & Total & 3.449 & 1.168 & 0.771 & 6.796 & 1.037 & 0.852 & 0.710 & 0.899 & 0.914 \\
\hline & & \multicolumn{3}{|c|}{$F P$} & \multicolumn{3}{|c|}{$F N$} & \multicolumn{3}{|c|}{$M B E[\mathrm{~mm}]$} \\
\hline & & no & r/a & ffd & no & r/a & ffd & no & $\mathbf{r} / \mathbf{a}$ & ffd \\
\hline \multirow{4}{*}{ 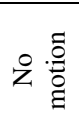 } & Basal & 0.092 & 0.092 & 0.071 & 0.024 & 0.024 & 0.050 & 0.042 & 0.042 & 0.024 \\
\hline & Medial & 0.079 & 0.079 & 0.086 & 0.078 & 0.079 & 0.097 & 0.008 & 0.009 & 0.005 \\
\hline & Apical & 0.127 & 0.127 & 0.122 & 0.062 & 0.062 & 0.102 & 0.003 & 0.003 & 0.013 \\
\hline & Total & 0.099 & 0.099 & 0.093 & 0.055 & 0.055 & 0.083 & 0.011 & 0.010 & 0.002 \\
\hline \multirow{4}{*}{ 在 } & Basal & 0.241 & 0.073 & 0.069 & 0.193 & 0.075 & 0.061 & 1.814 & 0.004 & 0.013 \\
\hline & Medial & 0.360 & 0.119 & 0.105 & 0.293 & 0.102 & 0.069 & 1.382 & 0.012 & 0.002 \\
\hline & Apical & 0.378 & 0.136 & 0.126 & 0.274 & 0.101 & 0.086 & 1.463 & 0.151 & 0.049 \\
\hline & Total & 0.326 & 0.109 & 0.100 & 0.253 & 0.093 & 0.072 & 1.553 & 0.056 & 0.021 \\
\hline
\end{tabular}

A quantitative evaluation was performed by manually delineating the left ventricle and myocardium. For every selected series, two single 2D slices were selected. One is the template and the other one is chosen when the myocardium motion was more discernible. For the subjects without significant motion, 5 series are selected for every slice position (basal, medial and apical), while for the group with significant motion a total of 30 series are used (10 for each slice position). As a result, a total of 45 series are randomly selected, covering all the different MR pulse sequences. Six statistical measures are computed to give a comprehensive quantification:

- $\boldsymbol{T}_{A-I}$ and $\boldsymbol{T}_{S-L}$ : the relative motion of the left ventricle center point between the template and the registered slice (A-I and S-L, Anterior-Inferior and Septal-Lateral directions);

- Dice ratio: the myocardium overlap ratio; 
- $\boldsymbol{F P}$ and $\boldsymbol{F N}$ : the false positive and false negative ratios of the myocardium mask;

- MBE: the myocardium boundary errors defined as the minimal distances between myocardium contours (endo and epi) extracted from the template and the registered slice.

\section{Results}

We applied the proposed method to all 586 MR perfusion series. As demonstrated in Fig. 3 where a typical example of MR perfusion motion compensation is given, with the effective rigid/affine and non-rigid registration, the jitter motion of myocardium can be largely eliminated. This corrected perfusion series will enable cardiologists to visually identify perfusion defects and further make the generation of perfusion parametric maps from the signal time course possible.

Qualitative evaluation revealed that for all 196 series without significant motion, no 'failed' cases were found and all series are graded as 'same', which means the motion correction did not introduce discernible errors. For 390 series with significant motion, 6 series $(\sim 1.5 \%)$ had large motion remaining after correction and were classified as 'not improved', while all others are noticeably improved. Again, no 'failed' cases were reported.

The results of qualitative assessment emphasize the robustness of proposed technique. The fact that no 'failed' cases were found proves proposed motion compensation scheme is highly reliably and can deal with variant MR pulse sequences with different SNR and myocardium contrast characteristics. Also, the registration does not show special preference for any specific slice position, which further strengthens its clinical applicability.

In all cases the non-rigid motion correction works well if the rigid/affine registration is successful. Table 1 summarizes the results from the quantitative measures before and after motion correction. For the series without significant motion, these measures show very small changes after the motion correction, while for the series with significant motion, the improvement is quite noticeable. Furthermore, the improvement from the non-rigid registration is discernible, but less significant if compared to the rigid/affine registration. This may be interpreted by the fact that

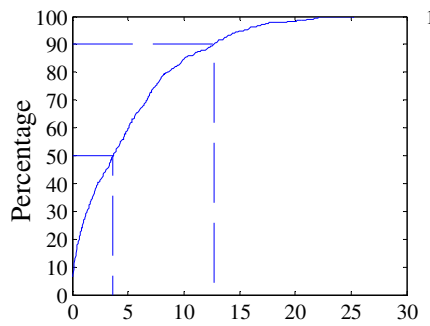

(a) $\boldsymbol{T}_{S-L}(\mathrm{~mm})$

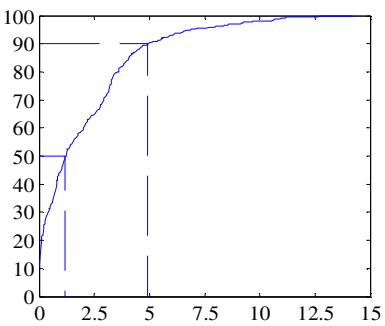

(b) $\boldsymbol{R}$ (degree)

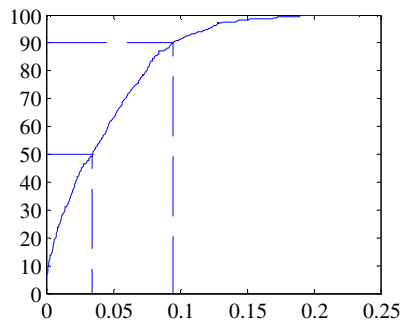

(c) $S$

Fig. 4. The accumulated distribution histograms over all perfusion series to reveal the maximal magnitude of possible myocardium motion 
myocardium motion in a perfusion series are mainly caused by the patient breathing along the septal-lateral direction.

Given the satisfied results of motion compensation and the considerable amount of perfusion series used for evaluation, we can perform a retrospective study to reveal the maximal magnitude of possible myocardium motion. Specifically, for every perfusion series, its maximal translation along the S-I direction $\boldsymbol{T}_{S-L}$, rotation $\boldsymbol{R}$ and scaling values $S$ were estimated from the rigid/affine registration. The accumulated distribution histograms of these measures are shown in Fig. 4. As shown in Fig. 4 (a), the $\boldsymbol{T}_{S-L}$ values of $90 \%$ of all perfusion series are less than $12.7 \mathrm{~mm}$, while the $50 \%$ of all series move for more than $3.7 \mathrm{~mm}$. Compared to the significant translation, Fig. 4(b) and (c) show the rotation and scaling of myocardium are less serious. The maximal rotations of $90 \%$ of all series are less than $5 \%$ and the scaling values are less than 0.1 . These statistics highlight the necessary minimal capture range for a robust MR perfusion motion compensation method.

\section{Discussion and Conclusions}

Analysis of a large collection of data obtained under clinically relevant conditions confirmed that motion correction is a frequent requirement (67\% of cases in this sample). As only highly reliable motion compensation approaches can be routinely used, we have developed a highly robust solution including both rigid/affine and the nonrigid image registration. The proposed method is tested on a large multi-center MR perfusion dataset covering typical clinical configurations. Both visual assessment and qualitative evaluation are performed and we have shown that our techniques are effective in $98.5 \%$ of the cases tested. The qualitative evaluation shows the non-rigid registration only slightly improves the motion compensation, which can lead to the questioning of its practical value in the clinical setting because of the heavy computational workload required. As a result, we would like to get more clinical feedback from professional cardiologists and improve the understanding of the role that the non-rigid registration may play in the context of MR perfusion motion compensation.

\section{References}

1. Puvaneswary, M.: Cardiac MR Imaging Anshan Ltd. (2005)

2. Canet, E.P., Janier, M.F., Revel, D.: Magnetic resonance perfusion imaging in ischemic heart disease. J. Magn. Reson. Imaging 10(3), 423-433 (1999)

3. Nagel, E., Klein, C., Paetsch, I., Hettwer, S., Schnackenburg, B., Wegscheider, K., Fleck, E.: Magnetic resonance perfusion measurements for the noninvasive detection of coronary artery disease. Circulation 108(4), 432-437 (2003)

4. Adluru, G., DiBella, E.V., Schabel, M.C.: Model-based registration for dynamic cardiac perfusion MRI. J. Magn. Reson. Imaging 24(5), 1062-1070 (2006)

5. Bidaut, L.M., Vallee, J.P.: Automated registration of dynamic MR images for the quantification of myocardial perfusion. J. Magn. Reson. Imaging 13(4), 648-655 (2001)

6. Bracoud, L., Vincen, F., Pachai, C., Canet, E., Croisille, P., Revel, D.: Automatic Registration of MR First-Pass Myocardial Perfusion Images. In: Magnin, I.E., Montagnat, J., Clarysse, P., Nenonen, J., Katila, T. (eds.) FIMH 2003. LNCS, vol. 2674, pp. 215-223. Springer, Heidelberg (2003) 
7. Comte, A., Lalande, A., Aho, S., Walker, P.M., Brunotte, F.: Realignment of myocardial first-pass MR perfusion images using an automatic detection of the heart-lung interface. Magn. Reson. Imaging 22(7), 1001-1009 (2004)

8. Dornier, C., Ivancevic, M.K., Thevenaz, P., Vallee, J.P.: Improvement in the quantification of myocardial perfusion using an automatic spline-based registration algorithm. J. Magn. Reson. Imaging 18(2), 160-168 (2003)

9. Wong, K., Wu, E., Ng, M., Wu, Y., Tse, H., Lau, C., Lo, G., Yang, E.: Image registration in myocardial perfusion MRI. Proc. IEEE Eng. Med. Biol. 1, 453-454 (2005)

10. Ablitt, N.A., Gao, J., Keegan, J., Stegger, L., Firmin, D.N., Yang, G.Z.: Predictive cardiac motion modeling and correction with partial least squares regression. IEEE Trans. Med. Imaging 23(10), 1315-1324 (2004)

11. Milles, J., van der Geest, R.J., Jerosch-Herold, M., Reiber, J.H., Lelieveldt, B.P.: Fully automated registration of first-pass myocardial perfusion MRI using independent component analysis. Inf. Process Med. Imaging 20, 544-555 (2007)

12. Yang, G.Z., Burger, P., Panting, J., Gatehouse, P.D., Rueckert, D., Pennell, D.J., Firmin, D.N.: Motion and deformation tracking for short-axis echo-planar myocardial perfusion imaging. Med. Image Anal. 2(3), 285-302 (1998)

13. Stegmann, M.B., Olafsdottir, H., Larsson, H.B.: Unsupervised motion-compensation of multi-slice cardiac perfusion MRI. Med. Image Anal. 9(4), 394-410 (2005)

14. Rueckert, D., Sonoda, L.I., Hayes, C., Hill, D.L., Leach, M.O., Hawkes, D.J.: Nonrigid registration using free-form deformations: application to breast MR images. IEEE Trans. Med. Imaging 18(8), 712-721 (1999)

15. Schnabel, J.A., Rueckert, D., Quist, M., Blackall, J.M., Castellano Smith, A.D., Hartkens, T., Penney, G.P., Hall, W.A., Liu, H., Truwit, C.L., Gerritsen, F.A., Hill, D.L.G., Hawkes, D.J.: A generic framework for non-rigid registration based on non-uniform multi-level free-form deformations. In: MICCAI, pp. 573-581 (2001)

16. Studholme, C., Hill, D.L., Hawkes, D.J.: Automated 3-D registration of MR and CT images of the head. Med. Image Anal. 1(2), 163-175 (1996) 